\title{
CATALYTIC ADVANTAGES PROVIDED BY SELENOCYSTEINE IN METHIONINE-S-SULFOXIDE REDUCTASES ${ }^{\dagger}$
}

\author{
Hwa-Young Kim $\ddagger$, Dmitri E. Fomenko $\ddagger$, Yeo-Eun Yoon $\ddagger, \S$, and Vadim N. Gladyshev $\ddagger$, \\ \$Department of Biochemistry, University of Nebraska, Lincoln, Nebraska 68588
}

$\S$ Dordt College, Sioux Center, Iowa 51250

\begin{abstract}
Methionine sulfoxide reductases are key enzymes that repair oxidatively damaged proteins. Two distinct stereospecific enzyme families are responsible for this function: MsrA (methionine- $S$ sulfoxide reductase) and $\mathrm{MsrB}$ (methionine- $R$-sulfoxide reductase). In the present study, we identified multiple selenoprotein MsrA sequences in organisms from bacteria to animals. We characterized the selenocysteine (Sec)-containing Chlamydomonas MsrA and found that this protein exhibited 10-50-fold higher activity than either its cysteine (Cys) mutant form or the natural mouse Cys-containing MsrA, making this selenoenzyme the most efficient MsrA known. We also generated a selenoprotein form of mouse MsrA and found that the presence of Sec increased the activity of this enzyme when a resolving Cys was mutated in the protein. These data suggest that the presence of Sec improves the reduction of methionine sulfoxide by MsrAs. However, the oxidized selenoprotein could not always be efficiently reduced to regenerate the active enzyme. Overall, this study demonstrates that sporadically evolved Sec-containing forms of methionine sulfoxide reductases reflect catalytic advantages provided by Sec in these and likely other thiol-dependent oxidoreductases.
\end{abstract}

Selenocysteine ( $\mathrm{Sec})^{1}$-containing proteins have been described in organisms from bacteria to humans. Over the past decade, significant progress has been made in identification and functional characterization of individual selenoproteins as well as entire sets of selenoproteins in organisms. These advances were possible due to the development of bioinformatics methods that allow efficient detection of selenoprotein genes in sequence databases (1-6). These genes have been identified by searching for a stem-loop structure in selenoprotein mRNAs, Sec insertion sequence (SECIS) element, as well as by SECIS-independent bioinformatics methods.

Methionine sulfoxides are generated by oxidation of methionine residues in proteins. However, these oxidized residues can be reversibly reduced back to methionine by repair enzymes, methionine sulfoxide reductases (7). These enzymes are represented by two distinct families: MsrA that is stereospecific for reduction of methionine- $S$-sulfoxide and MsrB specific for methionine- $R$-sulfoxide. A selenoprotein $\mathrm{R}$ (also known as selenoprotein $\mathrm{X}$ ) is the first mammalian selenoprotein identified by using bioinformatics methods $(1,2)$. Later, this

\footnotetext{
${ }^{\dagger}$ This study was supported by NIH AG021518 (to VNG). The computational part of this project was performed in part utilizing the Prairiefire Beowulf cluster from Research Computing Facility of the University of Nebraska-Lincoln, and the use of ${ }^{75}$ Se was supported by DoE DE-FG07-02ID14380.

*Corresponding author: N151 Beadle Center, Department of Biochemistry, University of Nebraska, Lincoln, Nebraska 68588; Tel, 402-472-4948; Fax, 402-472-7842; E-mail: vgladyshev1@unl.edu.

${ }^{1}$ Abbreviations: MsrA, methionine- $S$-sulfoxide reductase; MsrB, methionine- $R$-sulfoxide reductase; DTT, dithiothreitol; Sec, selenocysteine; SECIS, selenocysteine insertion sequence; Trx, thioredoxin.
} 
selenoprotein was found to function as MsrB $(8,9)$. In addition to this enzyme (MsrB1), two Cys-containing MsrBs (MsrB2 and MsrB3) have been found in mammals, in which Sec is replaced with Cys at the catalytic site $(10,11)$. Furthermore, a single selenoprotein MsrA has recently been detected in the green alga, Chlamydomonas reinhardtii, but this protein has not been characterized (12).

In previous studies, Sec was suspected to provide catalytic advantage for certain oxidoreductases (13). However, a study that examined Drosophila thioredoxin (Trx) reductase suggested that Sec is not necessary for high catalytic efficiency, but instead provides advantages with respect to broader substrate specificity and more flexible microenvironmental conditions in the active site (14). In our previous studies $(11,15)$, we characterized mammalian selenoprotein MsrBs and found that Sec plays an important role in MsrB enzyme activity. The naturally occurring selenoprotein MsrB1 has a 100-fold higher activity than its Cys mutant form. In addition, replacement of catalytic Cys with Sec results in more than 100-fold increased activities in MsrB2 and MsrB3 enzymes in dithiothreitol (DTT)-dependent reaction assays. These data suggested that higher catalytic activity per se is the advantage provided by Sec in MsrBs.

In the present study, we identified 14 selenoprotein MsrAs in organisms from bacteria to animals by searching for Sec/Cys pairs in predicted MsrA homologs. We also report the first functional characterization of selenoprotein MsrA. The data suggest that in the selenoprotein MsrA forms, as in selenoprotein MsrBs, Sec provides a key catalytic advantage.

\section{MATERIALS AND METHODS}

\section{Identification of selenoprotein MsrAs}

We detected Sec-containing MsrA by searching for Sec/Cys pairs in MsrA homologs $(4,5)$ in the following NCBI sequence databases: non-redundant protein, non-redundant nucleotide sequence, shotgun sequence, EST (all released Mar 9, 2006), conserved domains (Mar 4, 2006), taxonomy and environmental nucleotide sequence databases with accession numbers AACY00000000 (Dec 23, 2004) (16) AAFX01000000 (Feb 19, 2005), AAFY01000000 (Feb 23, 2005), AAFZ01000000 (Feb 23, 2005), AADL01000000 (May 05, 2004) (17), DU731018DU796676 and DU800850-DU800864 (Jan 27, 2006) (18).

We used blastall-program from stand alone BLAST package (19) with expectation value 0.01 for creating the initial set of MsrA proteins. The presence of MsrA domain (CDD 25795) was confirmed using RPS-BLAST and NCBI database of conserved domains. The resulting set of 357 proteins was filtered using NCBI taxonomy database to collect equal number of MsrA sequences from each life kingdom. Resulting protein sets were used for searching for Seccontaining MsrAs in various NCBI nucleotide sequence databases with tblastn program with the expectation value of 10. An in-house Perl script was used for automatic analysis of tblastn output to identify Cys residues aligned with candidate Sec. We selected sequences which were represented by at least two $\mathrm{Cys} / \mathrm{Sec}$ pairs from same organism as strong candidates to minimize the possibility of false positives due to sequencing errors, but all remaining sequences was also used in subsequent SECIS element prediction. Resulting sequence sets were manually analyzed for the presence of eukaryotic, archaeal and bacterial SECIS elements using SECISearch (4, 5) and RNAfold (20).

Sequence alignments were prepared using T-Coffee and colored in BoxShade 3.21 program. Sequence clustering was performed using ClustalW and ProtDist from Phylip package. The phylogenetic tree was prepared with Neighbor-joining method and visualized by PhyloDraw. 


\section{Cloning, expression, and purification of wild-type and mutant forms of Chlamydomonas MsrA}

A coding region of the selenoprotein MsrA gene was PCR-cloned into NdeI/XhoI sites of pET21b (Novagen) using cDNA libraries obtained from the Chlamydomonas Genetic Center, Duke University. To amplify the coding region, forward 5'-

ACACCATATGGCGACTAACGGGAACG-3' and reverse 5'-

AAAACTCGAGCCACCAGCCCGCAGAGCC-3' primers were used. The resulting plasmid was designated pET-CR-MsrA. This construct contained the full-length selenoprotein MsrA with a C-terminal His tag (LEHHHHH). We also generated a Cys mutant form in which Sec20 was replaced with Cys by site-directed mutagenesis. The resulting construct was named pETCR-MsrA/U20C.

To express Chlamydomonas selenoprotein MsrA in mammalian cells, we generated a GSTfused construct as follows. The full-length MsrA with a C-terminal His tag was amplified using pET-CR-MsrA as a template and cloned into EcoRI/SalI sites of pGEX-4T-1 (Amersham Biosciences). Then, the N-terminal GST-fused MsrA with a C-terminal His tag was PCRamplified and inserted into NheI/SalI sites of pCIMU-SECIS. This vector is derived from pCIneo (Promega) and contains a modified SECIS element of Toxoplasma gondii SelT (Novoselov and Gladyshev, unpublished). The resulting plasmid was named pCIMU-GST-CR-MsrA. Through site-directed mutagenesis, we generated several mutant constructs including U20C (a Cys mutant), C55S, C94S, and C55S/C94S. We also generated a construct, pGEX-CRMsrA/U20C, to express the GST-fused Cys mutant form with a C-terminal tag in E. coli. The full-length Cys mutant fragment with a C-terminal His tag was PCR-amplified from pET-CRMsrA/U20C and cloned into EcoRI/SalI sites of pGEX-4T-1. All constructs were verified by DNA sequencing.

To express wild-type and mutant forms of GST-fused Chlamydomonas MsrA in HEK293 cells, the constructs were separately transfected into the cells using a calcium phosphate method (21). To increase the expression levels of selenoprotein forms, a pCR3.1-SBP2 construct coding for rat SBP2 protein (22) was co-transfected. The transfected cells were cultured in Dulbecco's modified Eagle medium (DMEM) containing 10\% fetal calf serum, 0.15-0.6 $\mu \mathrm{M}$ sodium selenite, and antibiotics for $2-3$ days at $37^{\circ} \mathrm{C}$ under $5 \% \mathrm{CO}_{2}$. For comparison, the Cys mutant (U20C) was separately expressed in HEK293 cells. The GST-fused proteins with a Cterminal tag were purified using Talon metal-affinity resin (Clontech) as previously described (15). The eluted proteins were desalted and concentrated using Centricon YM-10 (Amersham Bioscience) with buffer A (50 mM sodium phosphate, $\mathrm{pH} 7.5$, and $50 \mathrm{mM} \mathrm{NaCl})$.

Unexpectedly, we found that endogenous MsrA protein had an affinity to the resin and was co-eluted. $200 \mathrm{ml}$ of HEK 293 cells was used ( 20 plates, $100 \mathrm{~mm}$ diameter) for transfection and the yield of purified selenoproteins was $2 \mu \mathrm{g}$ from the crude extracts containing $45 \mu \mathrm{g}$ of expressed selenoproteins (see supplementary Table 1).

The constructs that code for bacterially-expressed Cys mutant forms, pET-CR-MsrA/U20C (full-length) and pGEX-CR-MsrA/U20C (GST-fused), were expressed in E. coli BL21(DE3) cells. The proteins were purified using Talon metal-affinity resin and dialyzed against buffer A. The purified proteins were examined for purity by SDS-PAGE (supplementary Figure 1). The purified GST-fused protein was used for western blotting as an internal standard to determine the concentrations of GST-fused proteins in the transfected HEK293 cells and affinity enriched fractions. 


\section{Construction, expression, and purification of wild-type and selenoprotein forms of mouse MsrA}

Mouse MsrA(21-233), which lacks the mitochondrial signal peptide, was PCR-amplified using pET-MsrA(L)-His (23) as template and cloned into EcoRI/SalI sites of pGEX-4T-1, resulting in a plasmid pGEX-Mus-MsrA. This construct contained a C-terminal His tag (LEHHHHH). To generate a mammalian expression construct, the GST-fused MsrA fragment containing the C-terminal His tag was PCR-amplified using pGEX-Mus-MsrA as template and cloned into NheI/SalI sites of pCIMU-SECIS. Then, the catalytic Cys72 was replaced with Sec by sitedirect mutagenesis to generate a selenoprotein form. We also prepared C218S, C227S, or C218S/C227S mutants in the Cys- or Sec-containing forms.

Procedures for expression and purification of Cys- or Sec-containing forms from HEK 293 cells were as described above for Chlamydomonas MrrA.

The construct, pGEX-Mus-MsrA, was expressed in E. coli BL21(DE3) cells, purified using Talon metal-affinity resin, and used as an internal standard for western blotting to determine the concentrations of proteins from the transfected HEK293 cells.

\section{Metabolic labeling with ${ }^{75} \mathrm{Se}$}

Transfections of constructs coding for GST-fused Chlamydomonas MsrA and GST-fused mouse selenoprotein MsrA into NIH 3T3 cells were carried out using Lifopectamine and Plus reagents (Invitrogen) according to the manufacturer's instructions. The transfected cells were grown for $24 \mathrm{~h}$ in DMEM containing $10 \mu \mathrm{Ci} / \mathrm{ml}$ of ${ }^{75} \mathrm{Se}$ (Research Reactor Facility, University of Missouri, Columbia, MO), 10\% fetal calf serum, and antibiotics. Cells were washed with PBS buffer and suspended in CelLytic-M (Sigma) lysis buffer. ${ }^{75}$ Se labeled selenoproteins were resolved by SDS-PAGE and detected using a PhosphorImager (Amersham).

\section{Determination of protein concentration}

Concentration of purified proteins expressed in E. coli was determined by Bradford method using a Bio-Rad protein assay reagent (Bio-Rad). Bovine serum albumin was used as a standard. Concentration of proteins expressed in HEK 293 cells was determined by western blotting using specific antibodies and bacterially-expressed proteins as internal standards, followed by quantifying the western blot signals with a densitometer. In the western blots of GST-fused Chlamydomonas MsrAs, anti-GST antibodies (Amersham Biosciences) were used. Anti-GST or anti-MsrA antibodies were used for the detection of GST-fused mouse MsrAs, and anti-MsrA antibodies for the endogenous MsrA.

\section{MsrA enzyme assay and analysis of kinetics}

MsrA activity was measured in the presence of DTT or Trx. In the DTT-dependent reaction, a $100 \mu \mathrm{l}$ reaction mixture contained $50 \mathrm{mM}$ sodium phosphate, $\mathrm{pH} 7.5,50 \mathrm{mM} \mathrm{NaCl}, 20 \mathrm{mM}$ DTT, $200 \mu \mathrm{M}$ dabsyl-methionine-S-sulfoxide, and purified (or crude) proteins. In the Trxdependent reaction, the reaction mixture $(100 \mu \mathrm{l})$ contained $50 \mathrm{mM}$ sodium phosphate, $\mathrm{pH} 7.5$, $50 \mathrm{mM} \mathrm{NaCl}, 0.2 \mathrm{mM}$ NADPH, $6.8 \mu \mathrm{M}$ E. coli $\mathrm{Trx}$ (Sigma), $0.4 \mu \mathrm{M}$ E. coli Trx reductase (Sigma), $200 \mu \mathrm{M}$ dabsyl- methionine-S-sulfoxide, and purified (or crude) proteins. The reactions were carried out at $37^{\circ} \mathrm{C}$ for $30 \mathrm{~min}$ and analyzed by HPLC as described previously (24). The enzyme activity of purified proteins from transfected HEK 293 cells was calculated by subtracting endogenous MsrA activity from total activity because the samples contained a smallportion of the endogenous MsrA. The endogenous MsrA activity from transfected cells was normalized to the activity in mock-transfected HEK 293 cells as internal control.

Contaminating MsrA activity observed in the purified samples varied depending upon expressed proteins and reactions. To make sure our data are solid, we had to carefully quantify 
the contaminating activity. The contaminating MsrA activity was as follows: $2 \%$ of total DTTdependent activity and 38\% of total Trx-dependent activity in purified GST-fused Chlamydomonas wild-type proteins, 5\% of total DTT-dependent activity and 40\% of total Trxdependent activity in purified GST-fused Chlamydomonas U20C proteins, 3\% of total DTTor Trx-dependent activities in purified GST-fused mouse wild-type proteins, and $48 \%$ of total DTT-dependent activity and 34\% of total Trx-dependent activity in purified GST-fused mouse C72U proteins. The endogenous MsrA activity from crude protein extracts was not detectable in our assay conditions where $100-150 \mu \mathrm{g}$ of crude protein was used.

$K_{\mathrm{m}}$ and $k_{\mathrm{cat}}$ values were determined from Lineweaver-Burk plots using 50-800 $\mu \mathrm{M}$ substrate in the DTT-dependent reaction (supplementary Figure 2).

\section{Determination of molecular mass}

Apparent molecular mass of Chlamydomonas MsrA and its derivatives was determined by gel filtration using a TSK-GEL G3000PWXL column (Tosoh Bioscience) and a Superdex 200 column (Amersham Biosciences). The recombinant Cys mutant proteins purified from $E$. coli were loaded onto the column and eluted with PBS buffer (TSK-GEL G3000PWXL column) or with $50 \mathrm{mM}$ Tris-HCl, pH 8.0, and $100 \mathrm{mM} \mathrm{KCl}$ (Superdex 200 column). The fulllength form was found to migrate as a monomer, but the GST-fused form was determined to be a dimer. Gel filtration standards purchased from Bio-Rad were used as molecular weight markers.

\section{RESULTS AND DISCUSSION}

\section{Identification of selenoprotein MsrAs and evolutionary implications}

MsrAs are present in most organisms with the exception of some intracellular parasites and hyperthermophiles. We analyzed NCBI nucleotide sequence databases using the sequence alignment method of searching for Sec/Cys pairs in MsrA sequences (see Material and Methods) and identified 14 Sec-containing MsrA homologs (gi 23452038, 70062517, 34792792, 89467751, 66511346, 89138292, 51995432, 49561997, 58079970, 21640047, 63523529, 68769619, 71547479, 66861845; 13 from eukaryotes and 1 from bacterium Syntrophobacter fumaroxidans) (Figure 1A). Among 13 eukaryotic Sec-containing MsrAs, three were from algae (C. reinhardtii, Bigelowiella natans, Ostreococcus tauri), four were tick sequences (Boophilus microplus, Amblyomma variegatum, A. americanum, Ixodes scapularis), one was a spider homolog (Acanthoscurria gomesiana) and five sequences were from different aquatic organisms (Hydra magnipapillata, Strongylocentrotus purpuratus, Paracentrotus lividus, Acropora millepora, Branchiostoma floridae). No selenoprotein MsrAs were detected in vertebrate animals and archaea. This distribution contrasted with that of selenoprotein MsrBs which previously were identified only in vertebrates. The detected eukaryotic selenoprotein MsrAs contained typical eukaryotic SECIS elements in their 3-UTRs, whereas the bacterial $S$. fumaroxidans sequence had a typical bacterial SECIS element in the coding region immediately downstream of Sec-encoding UGA codon (Figure 1B).

Multiple sequence alignment of Sec- and Cys-containing MsrAs was constructed to examine the sequences for motifs and/or residues uniquely conserved in selenoprotein MsrAs, but no such sequences could be found. Phylogenetic analysis revealed that the majority of Seccontaining sequences clustered together except for three algal and one bacterial proteins (Figure 1C). The cluster of Sec-containing proteins also included insect Cys-containing MsrAs. Cyscontaining MsrAs occur in most organisms, but only a small subset of these proteins are selenoproteins, suggesting that the Sec-containing forms evolved from Cys-containing homologs. It is unclear whether most of the Cys-to-Sec replacements occurred sporadically or were the result of a limited number of events followed by speciation and/or horizontal gene 
transfer. However, the observed diversity of selenoprotein MsrAs clearly favors multiple independent events.

\section{Characterization of Chlamydomonas selenoprotein MsrA}

The detection of Sec-containing MsrA homologs does not necessarily mean that these proteins are functional methionine sulfoxide reductases. To functionally characterize Sec-containing MsrAs, we amplified a coding region of selenoprotein MsrA from a C. reinhardtii cDNA library (see Materials and Methods). The cloned sequence coded for a protein of 160 residues and had 4 single nucleotide differences (corresponding to Lys30, Leu31, Gln107 an Arg108) compared to the previously reported cDNA sequence (accession number AF494053). However, the cloned nucleotide sequence matched the Chlamydomonas genomic sequence. The Sec residue was located in the conserved catalytic motif, Gly19-Sec20-Phe21-Trp22 with Sec replacing the catalytic Cys in Cys-containing MsrAs.

To investigate catalytic properties of Chlamydomonas MsrA, we expressed a GST-fused form of this protein (e.g., GST-MsrA) in mammalian cells. To support Sec insertion, the construct also had a eukaryotic SECIS element in the 3'-UTR. Sec incorporation into this GST-fusion protein was confirmed by ${ }^{75} \mathrm{Se}$ metabolic labeling of NIH $3 \mathrm{~T} 3$ cells transfected with this construct (Figure 2A). We also carried out a large scale transfection in HEK 293 cells and then purified the GST-fused selenoprotein by affinity chromatography. For comparison, we separately expressed and purified a Cys mutant (U20C) of Chlamydomonas MsrA, also in HEK 293 cells. As shown in Table 1, the specific activity of the Chlamydomonas selenoprotein MsrA (GST-WT) was $5328 \mathrm{nmol} / \mathrm{min} / \mathrm{mg}$ protein in a DTT-dependent reaction. The selenoprotein was 10-fold more active compared to its Cys mutant (GST-U20C), suggesting a key contribution of selenium to the catalytic function of this enzyme. In addition, this selenoprotein had a 50-fold higher enzyme activity than the mouse Cys-containing GST-MsrA (see Table 2), a 25-fold higher activity than Drosophila MsrA (24), and a 100-fold higher activity than yeast MsrA (25). In fact, this selenoprotein appears to be the most efficient MsrA catalyst known. On the other hand, the activity of the Chlamydomonas selenoprotein was 20 -fold lower in a Trx-dependent reaction compared to the reaction carried out in the presence of DTT. The lower activity in presence of Trx was also observed for the Cys mutant expressed in either HEK 293 or E. coli cells.

We recently reported that a Cys mutant form of mouse selenoprotein MsrB1 had over 100-fold lower activity than the naturally occurring Cys-containing MsrB2 and MsrB3 (11). In contrast, the Cys mutant of Chlamydomonas MsrA had a high activity and was a superior catalyst compared even to the native mouse Cys-containing MsrA (Tables 1 and 2). There are three residues that appear to be unique for Cys-containing MsrBs and these amino acids were required for maximal activity of each isozyme (15). Similarly, we analyzed the sequences of Sec- and Cys-containing MsrAs and modeled the conserved residues on bovine and E. coli MsrAs, which are family members with known structures $(26,27)$. However, in contrast to MsrBs, this procedure did not result in additional insights into differences between Sec- and Cys-containing MsrAs. The active sites of both enzyme types had conserved Tyr51, Glu62, Tyr96, His143, and Tyr146 (numbering is based on the Chlamydomonas MsrA sequence), in addition to the catalytic motif containing catalytic Sec20 or corresponding Cys residues.

Although MsrA and MsrB have different structural folds (26-28), their reaction mechanisms are known to be similar (28-31) and include three steps. First, the catalytic Cys (or Sec) attacks the sulfoxide moiety of the substrate and itself is converted to a sulfenic acid (or selenenic acid) intermediate with concomitant release of the product, methionine. Second, the sulfenic acid (or selenenic acid) intermediate forms an intramolecular disulfide (or selenenylsulfide) bond with another Cys (or sometimes through a disulfide relay), which is known as the resolving Cys. Finally, the disulfide (or selenenylsulfide) bond is reduced by a reductant (Trx in vivo or 
DTT in vitro) to regenerate the active state. However, we recently reported that the sulfenic intermediate in human MsrB3 is also directly reducible by Trx, whereas the resolving Cys is needed for the Trx-dependent reduction of the intermediate in selenoprotein MsrB1 (15).

The resolving Cys residue of MsrA is located in the C-terminal region as shown for many enzymes (see Figures 1A and 3), such as those from mammals (26), E. coli (27), and Mycobacterium tuberculosis (32). Amino acid sequence analysis revealed that there are two Cys residues in the Chlamydomonas MsrA. These residues are located in the middle of the protein sequence (Cys55 and Cys94). Cys55 is conserved in some Cys-containing MsrAs, whereas Cys94 is not (Figure 1A). To test if Cys55 or Cys94 function as resolving Cys, we mutated these residues to Ser in the GST-fused MsrA to generate single and double mutants. As shown in Table 3, in the DTT-dependent assay, all single and double mutants were active, although C55S and C55S/C94S mutants exhibited 2-fold lower activities than the wild-type. In addition, all mutant proteins showed similar activities compared to the wild-type in the Trxdependent assay, suggesting that Cys55 and Cys94 do not serve as resolving Cys. Therefore, resolving Cys is absent in Chlamydomonas Sec-containing MsrA. No requirement for resolving Cys was also observed in the Cys mutant (U20C) of this enzyme (data not shown).

\section{Selenenylsulfide in MsrA is not efficiently reducible by DTT}

To test if insertion of Sec can provide catalytic advantage to Cys-containing MsrAs, we generated a selenoprotein form of mouse MsrA by replacing the catalytic Cys 72 with Sec in the GST-MsrA fusion protein. We confirmed Sec insertion into this protein by metabolic labeling of transfected cells with ${ }^{75} \mathrm{Se}$ (Figure $2 \mathrm{~B}$ ). We separately expressed in a large scale and purified the mutant selenoprotein and the wild type Cys-containing mouse MsrA fused to GST from transfected HEK 293 cells. Surprisingly, the activity of the selenoprotein form (GSTMus-C72U) was similar to that of the Cys-containing protein (GST-Mus-WT) in both DTTand Trx-dependent assays (Table 2).

In many selenoproteins, including Chlamydomonas MsrA in the present study, the use of Sec manifests a $10-100$ fold increased enzyme activity $(15,33)$. Why did not the selenoprotein form of mouse MsrA result in increased activity? In the previous study (15), we generated selenoprotein forms of MsrB2 and MsrB3, which lacked resolving Cys residues. These selenoproteins showed $>100$-fold increased activity in the DTT-dependent assay, but these proteins were not active in the presence of Trx. In an effort to restore the Trx-dependent activity, we inserted an artificial resolving Cys in the selenoprotein form of MsrB3. Although this protein recovered the Trx-dependent activity similarly to that of the wild-type Cys-containing form, its DTT-dependent activity was 16-fold lower compared to the original enzyme form without the resolving Cys. On the other hand, the resolving Cys was required for the activity of selenoprotein MsrB1 in the Trx-dependent assay but not in the DTT-dependent reaction.

Based on these previous observations, we hypothesized that the selenenic acid intermediate itself would be better reducible by DTT than the selenenylsulfide bond formed by Sec with the resolving Cys. Thus, we predicted that the removal of the resolving Cys would increase the DTT-dependent activity but impair the Trx-dependent activity of the Sec-containing mouse MsrA form.

To test this hypothesis, we generated C218S, C227S, and C218S/C227S mutants in both Cysand Sec-containing mouse MsrA forms. Cys218 is known to form a disulfide bond with the catalytic Cys in mouse MsrA, whereas Cys227 subsequently forms a disulfide bond with Cys218 releasing reduced catalytic Cys. The Cys218-C227 disulfide is then reduced by Trx or DTT $(26,29)$. As shown in Table 4, C218S of Cys-containing mouse MsrA had a severely impaired activity in both DTT and Trx assays (6\% and 7\% of the wild-type activity, respectively), indicating an important role of the resolving Cys in the catalytic function of this 
enzyme. The C228S mutant exhibited a slightly increased activity in the DTT assay, but had only $12 \%$ of the wild-type activity in the Trx assay. The activity of the double C218S/C227S mutant was $25 \%$ in the DTT assay and 3\% in the Trx reaction as compared to the corresponding activities of the wild-type enzyme. These effects were consistent with a previous report that described the role of resolving Cys in bovine MsrA (29). In contrast, the C218S mutant of Seccontaining mouse MsrA had an increased activity (170\% of the Sec-containing form containing the resolving Cys). Taken into account a combination of the 17 -fold decreased activity of the Cys-containing form and the 1.7-fold increased activity of the Sec-containing form, this C218S selenoprotein was calculated to be 29-fold more active than the corresponding Cys-containing protein. The C227S mutation resulted in a slightly increased activity in the DTT assay. The double mutant C218S/C227S exhibited a 3-fold increased activity in the presence of DTT. Thus, these data supported our hypothesis that the selenenylsulfide bond is not an efficient substrate for DTT as compared to the selenenic acid intermediate. The inefficient regeneration of MsrA in which Sec replaces Cys is likely due to the low redox potential of the selenenylsulfide intermediate. On the other hand, in the Trx assay, the C218S mutation severely affected the activity of the selenoprotein form, suggesting a requirement for the resolving Cys for the Trx-dependent activity.

\section{Similar catalytic mechanisms, advantages and disadvantages provided by Sec in MsrA and MsrB}

Replacement of Cys with Sec in MsrB2 and MsrB3 resulted in more than 100-fold higher activities of the enzymes (15). This higher activity occurs when the selenenic acid intermediate is a substrate for DTT. However, the use of Sec also causes catalytic disadvantages as the intermediate is not reducible by Trx, a natural reductant. In the present study, we found that similar catalytic advantages and disadvantages provided by Sec are also seen in the selenoprotein form of mouse MsrA. The selenenic acid intermediate was a much better substrate for DTT than the selenenylsulfide. In fact, if the resolving Cys is removed (preventing selenenylsulfide bond formation), the DTT-dependent activity was significantly increased. A high catalytic activity is an obvious catalytic advantage provided by Sec and the best illustration of this effect is Chlamydomonas MsrA - the most efficient MsrA known.

It should be noted that the Chlamydomonas MsrA activity was dramatically lower (>20-fold) when Trx rather than DTT was used as reductant. However, in the case of mouse MsrA, its Trx-dependent activity was 55\% of the DTT-dependent activity (Table 2). Moreover, all artificial or native selenoprotein forms tested (MsrB1, MsrB2, MsrB3, and mouse MsrA) except for the Chlamydomonas MsrA required the resolving Cys in the Trx-dependent assay. Our data argue that Trx may not be a natural reductant for Chlamydomonas MsrA. It is possible that there is an unknown reductant (i.e., a Trx-like protein or a dithiol compound) that is used in vivo to regenerate the active state of the enzyme.

\section{CONCLUSION}

In the present study, we detected a number of selenoprotein MsrAs in bacteria, algae and invertebrate animals. Interestingly, this wide distribution of Sec-containing MsrAs contrasted with the occurrence of selenoprotein MsrBs that were detected thus far only in vertebrates. In addition, we report the first functional characterization of any selenoprotein MsrA. The Seccontaining Chlamydomonas MsrA exhibited 10- to 50-fold higher activities than either its Cys mutant or natural Cys-containing MsrAs and is the most efficient MsrA known. Introducing Sec into mouse MsrA also increased the activity of this enzyme when the resolving Cys was removed and DTT was used as reductant. Even though MsrA and MsrB are structurally unrelated enzymes, not only their reaction mechanisms, but also the sporadic occurrence of Sec and catalytic advantages and disadvantages provided by this residue are similar in these 
enzyme families. Overall, our study provided evidence for catalytic advantages provided by Sec in thiol-dependent oxidoreductases, and also suggested why these advantages are not universally employed in nature.

\section{Supplementary Material}

Refer to Web version on PubMed Central for supplementary material.

\section{Acknowledgements}

The authors thank Dr. Sergey Novoselov for providing a construct with a eukaryotic SECIS element and helpful comments on selenoprotein expression.

\section{References}

1. Lescure A, Gautheret D, Carbon P, Krol A. Novel selenoproteins identified in silico and in vivo by using a conserved RNA structural motif. J Biol Chem 1999;274:38147-38154. [PubMed: 10608886]

2. Kryukov GV, Kryukov VM, Gladyshev VN. New mammalian selenocysteine-containing proteins identified with an algorithm that searches for selenocysteine insertion sequence elements. J Biol Chem 1999;274:33888-33897. [PubMed: 10567350]

3. Castellano S, Morozova N, Morey M, Berry MJ, Serras F, Corominas M, Guigó R. In silico identification of novel selenoproteins in the Drosophila melanogaster genome. EMBO Rep 2001;2:697-702. [PubMed: 11493597]

4. Kryukov GV, Castellano S, Novoselov SV, Lobanov AV, Zehtab O, Guigó R, Gladyshev VN. Characterization of mammalian selenoproteomes. Science 2003;300:1439-1443. [PubMed: 12775843]

5. Kryukov GV, Gladyshev VN. The prokaryotic selenoproteome. EMBO Rep 2004;5:538-543. [PubMed: 15105824]

6. Castellano S, Novoselov SV, Kryukov GV, Lescure A, Blanco E, Krol A, Gladyshev VN, Guigó R. Reconsidering the evolution of eukaryotic selenoproteins: a novel nonmammalian family with scattered phylogenetic distribution. EMBO Rep 2004;5:71-77. [PubMed: 14710190]

7. Weissbach H, Etienne F, Hoshi T, Heinemann SH, Lowther WT, Matthews B, St John G, Nathan C, Brot N. Peptide methionine sulfoxide reductase: structure, mechanism of action, and biological function. Arch Biochem Biophys 2002;397:172-178. [PubMed: 11795868]

8. Kryukov GV, Kumar RA, Koc A, Sun Z, Gladyshev VN. Selenoprotein R is a zinc-containing stereospecific methionine sulfoxide reductase. Proc Natl Acad Sci USA 2002;99:4245-4250. [PubMed: 11929995]

9. Moskovitz J, Singh VK, Requena J, Wilkinson BJ, Jayaswal RK, Stadtman ER. Purification and characterization of methionine sulfoxide reductases from mouse and Staphylococcus aureus and their substrate stereospecificity. Biochem Biophys Res Commun 2002;290:62-65. [PubMed: 11779133]

10. Jung S, Hansel A, Kasperczyk H, Hoshi T, Heinemann SH. Activity, tissue distribution and sitedirected mutagenesis of a human peptide methionine sulfoxide reductase of type B: hCBS1. FEBS Lett 2002;527:91-94. [PubMed: 12220640]

11. Kim HY, Gladyshev VN. Methionine sulfoxide reduction in mammals: characterization of methionine- $R$-sulfoxide reductases. Mol Biol Cell 2004;15:1055-1064. [PubMed: 14699060]

12. Novoselov SV, Rao M, Onoshko NV, Zhi H, Kryukov GV, Xiang Y, Weeks DP, Hatfield DL, Gladyshev VN. Selenoproteins and selenocysteine insertion system in the model plant cell system, Chlamydomonas reinhardtii. EMBO J 2002;21:3681-3693. [PubMed: 12110581]

13. Stadtman TC. Selenocysteine. Annu Rev Biochem 1996;65:83-100. [PubMed: 8811175]

14. Gromer S, Johansson L, Bauer H, Arscott LD, Rauch S, Ballou DP, Williams CH Jr, Schirmer RH, Arnér ES. Active sites of thioredoxin reductases: why selenoproteins? Proc Natl Acad Sci USA 2003;100:12618-12623. [PubMed: 14569031]

15. Kim HY, Gladyshev VN. Different catalytic mechanisms in mammalian selenocysteine- and cysteinecontaining methionine- $R$-sulfoxide reductases. PLoS Biol 2005;3:e375. [PubMed: 16262444] 
16. Venter JC, Remington K, Heidelberg JF, Halpern AL, Rusch D, Eisen JA, Wu D, Paulsen I, Nelson KE, Nelson W, Fouts DE, Levy S, Knap AH, Lomas MW, Nealson K, White O, Peterson J, Hoffman J, Parsons R, Baden-Tillson H, Pfannkoch C, Rogers YH, Smith HO. Environmental genome shotgun sequencing of the Sargasso Sea. Science 2004;304:66-74. [PubMed: 15001713]

17. Tringe SG, von Mering C, Kobayashi A, Salamov AA, Chen K, Chang HW, Podar M, Short JM, Mathur EJ, Detter JC, Bork P, Hugenholtz P, Rubin EM. Comparative metagenomics of microbial communities. Science 2005;308:554-557. [PubMed: 15845853]

18. DeLong EF, Preston CM, Mincer T, Rich V, Hallam SJ, Frigaard NU, Martinez A, Sullivan MB, Edwards R, Brito BR, Chisholm SW, Karl DM. Community genomics among stratified microbial assemblages in the ocean's interior. Science 2006;311:496-503. [PubMed: 16439655]

19. Altschul SF, Madden TL, Schaffer AA, Zhang J, Zhang Z, Miller W, Lipman D. Gapped BLAST and PSI-BLAST: a new generation of protein database search programs. Nucleic Acids Res 1997;25:3389-3402. [PubMed: 9254694]

20. Hofacker IL, Fontana W, Stadler PF, Bonhoeffer S, Tacker M, Schuster P. Fast folding and comparison of RNA secondary structures. Monatshefte f Chemie 1994;125:167-188.

21. Sambrook, J.; Fritsch, EF.; Maniatis, T. Molecular Cloning: A Laboratory Manual. 2. Cold Spring Harbor Laboratory Press; New York, USA: 1989.

22. Copeland PR, Fletcher JE, Carlson BA, Hatfield DL, Driscoll DM. A novel RNA binding protein, SBP2, is required for the translation of mammalian selenoprotein mRNAs. EMBO J 2000;17:306314. [PubMed: 10637234]

23. Kim HY, Gladyshev VN. Role of structural and functional elements of mouse methionine- $S$-sulfoxide reductase in its subcellular distribution. Biochemistry 2005;44:8059-8067. [PubMed: 15924425]

24. Kumar RA, Koc A, Cerny RL, Gladyshev VN. Reaction mechanism, evolutionary analysis, and role of zinc in Drosophila methionine- $R$-sulfoxide reductase. J Biol Chem 2002;277:37527-37535. [PubMed: 12145281]

25. Koc A, Gasch AP, Rutherford JC, Kim HY, Gladyshev VN. Methionine sulfoxide reductase regulation of yeast lifespan reveals reactive oxygen species-dependent and -independent components of aging. Proc Natl Acad Sci USA 2004;101:7999-8004. [PubMed: 15141092]

26. Lowther WT, Brot N, Weissbach H, Matthews BW. Structure and mechanism of peptide methionine sulfoxide reductase, an "anti-oxidation" enzyme. Biochemistry 2000;39:13307-13312. [PubMed: 11063566]

27. Tête-Favier F, Cobessi D, Boschi-Muller S, Azza S, Branlant G, Aubry A. Crystal structure of the Escherichia coli peptide methionine sulphoxide reductase at $1.9 \AA$ resolution. Structure 2000;8:1167-1178. [PubMed: 11080639]

28. Lowther WT, Weissbach H, Etienne F, Brot N, Matthews BW. The mirrored methionine sulfoxide reductases of Neisseria gonorrhoeae pilB. Nat Struct Biol 2002;9:348-352. [PubMed: 11938352]

29. Lowther WT, Brot N, Weissbach H, Honek JF, Matthews BW. Thiol-disulfide exchange is involved in the catalytic mechanism of peptide methionine sulfoxide reductase. Proc Natl Acad Sci USA 2000;97:463-6468.

30. Antoine M, Boschi-Muller S, Branlant G. Kinetic characterization of the chemical steps involved in the catalytic mechanism of methionine sulfoxide reductase A from Neisseria meningitides. J Biol Chem 2003;278:45352-45357. [PubMed: 12954610]

31. Olry A, Boschi-Muller S, Branlant G. Kinetic characterization of the catalytic mechanism of methionine sulfoxide reductase B from Neisseria meningitidis. Biochemistry 2004;43:11616-11622. [PubMed: 15350148]

32. Taylor AB, Benglis DM Jr, Dhandayuthapani S, Hart PJ. Structure of Mycobacterium tuberculosis methionine sulfoxide reductase A in complex with protein-bound methionine. J Bacteriol 2003;185:4119-4126. [PubMed: 12837786]

33. Hazebrouck S, Camoin L, Faltin Z, Strosberg AD, Eshdat Y. Substituting selenocysteine for catalytic cysteine 41 enhances enzymatic activity of plant phospholipid hydroperoxide glutathione peroxidase expressed in Escherichia coli. J Biol Chem 2000;275:28715-28721. [PubMed: 10874045] 
A

23452038 C. reinhardtii

70062517 O. tauri

34792792 s. purpuratus

89467751 P. lividus

66511346 B. floridae

89138292 A. millepora

$51995432 \mathrm{H}$. magnipapillata

49561997 B. microplus

$58079970 \mathrm{~A}$. americanum

21640047 A. variegatum

63523529 I. scapularis

68769619 A. gomesiana

71547479 S. fumaroxidans

66861845 B. natans

$23338220 \mathrm{D}$. melanogaster

55234261 A. gambiae

33089114 A. mellifera

39582354 C. briggsae

76258265 C. aurantiacus

$66799168 \mathrm{D}$. geothermalis

$32477189 R$. baltica

88933851 Dehalococcoides sp.

78171127 C. chlorochromatis

51470621 A. punctata

27806537 B. taurus

$33150552 \mathrm{H}$. sapiens

31981013 M. musculus

55251370 D. rerio

78709015 o. sativa

$7580480 \mathrm{~L}$. esculentum

21593143 A. thaliana

1136793 B. napus

29469000 G. barbadense

23452038 C. reinhardtii

70062517 O. tauri

$34792792 \mathrm{~S}$. purpuratus

89467751 P. lividus

66511346 B. floridae

89138292 A. millepora

51995432 H. magnipapillata

49561997 B. microplus

58079970 A. americanum

21640047 A. variegatum

63523529 I. scapularis

68769619 A. gomesiana

$71547479 \mathrm{~s}$. fumaroxidans

66861845 B. natans

23338220 D. melanogaster

$55234261 \mathrm{~A}$. gambiae

33089114 A. mellifera

39582354 C. briggsae

76258265 C. aurantiacus

$66799168 \mathrm{D}$. geothermalis

$32477189 R$. baltica

88933851 Dehalococcoides sp.

78171127 C. chlorochromatii

51470621 A. punctata

27806537 B. taurus

$33150552 \mathrm{H}$. sapiens

31981013 M. musculus

55251370 D. rerio

78709015 o. sativa

7580480 L. esculentum

21593143 A. thaliana

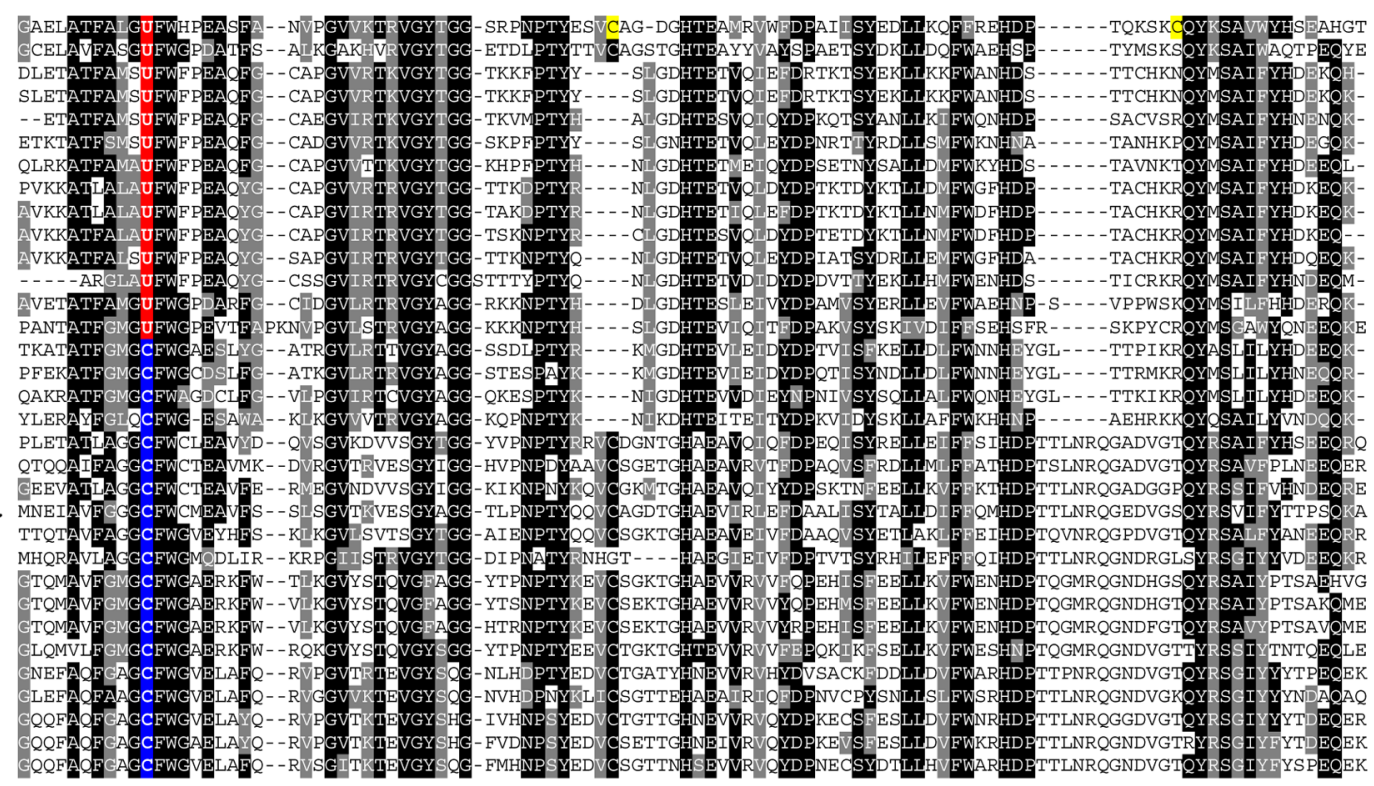

1136793 B. napus

29469000 G. barbadense

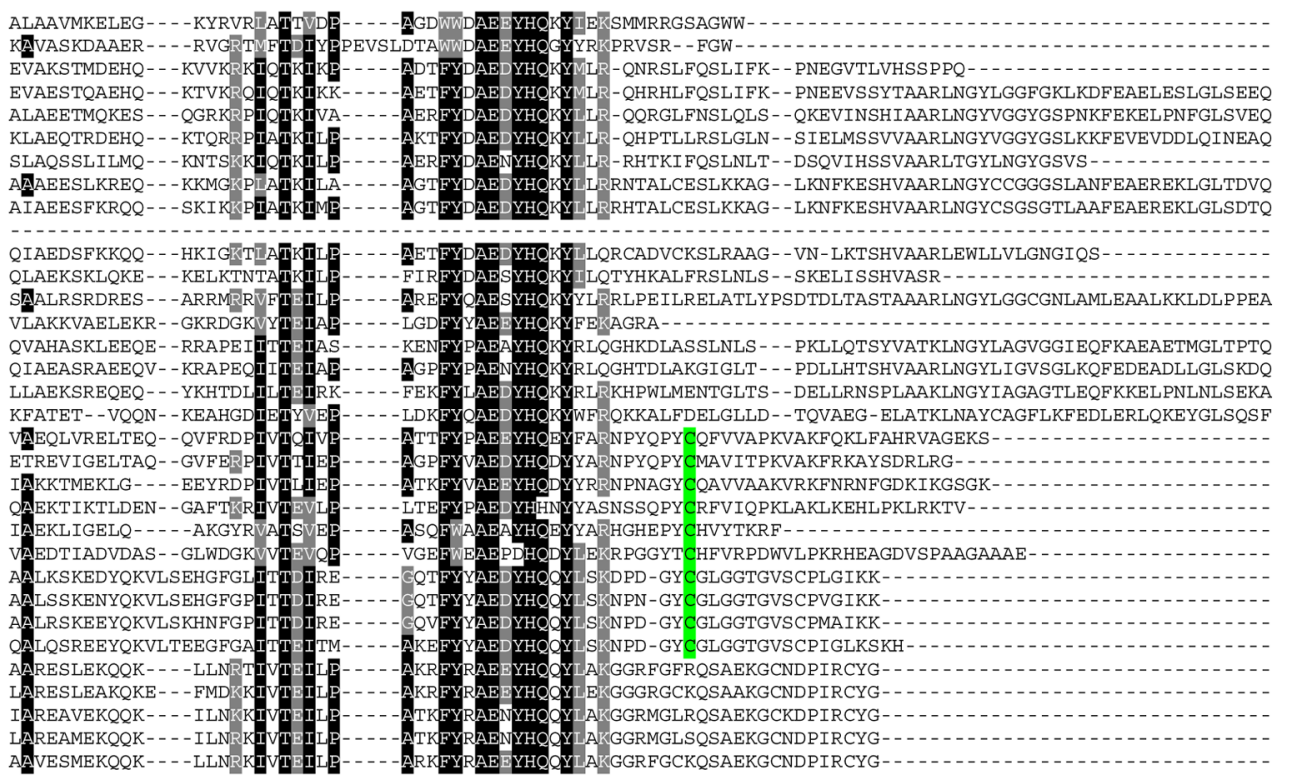



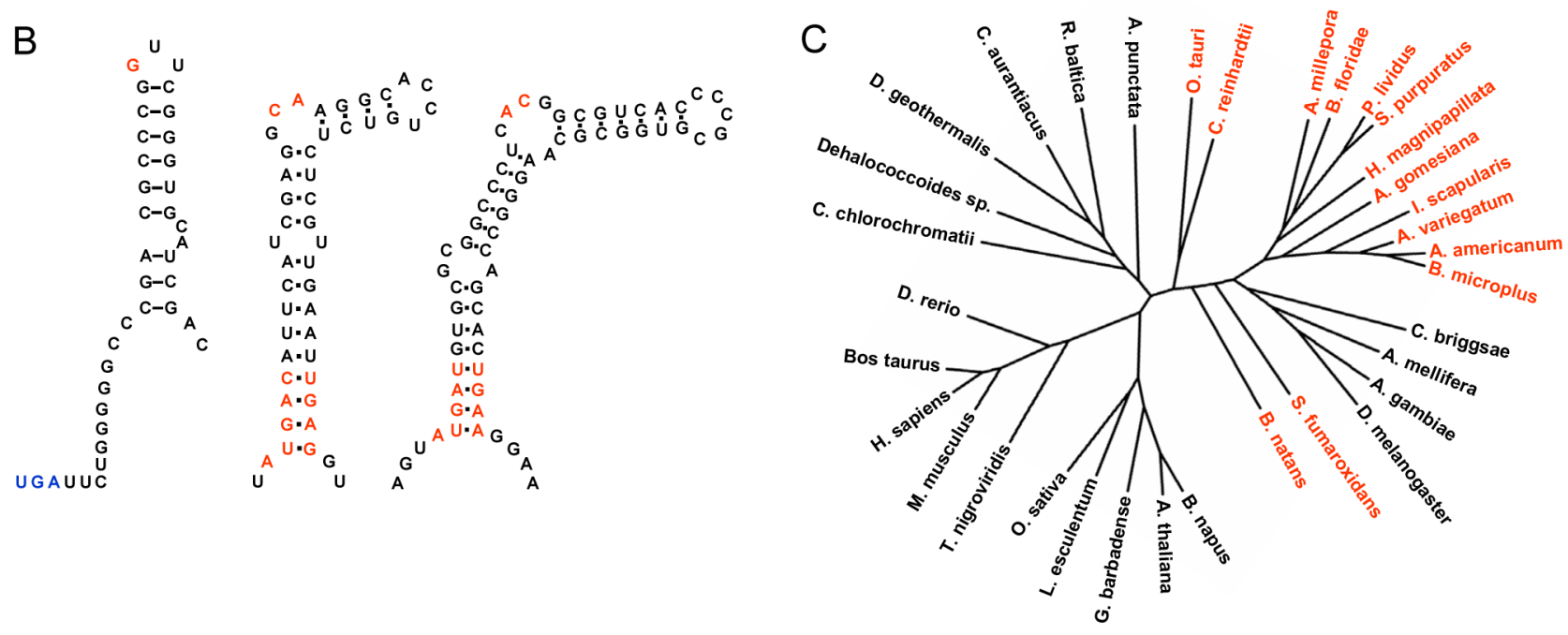

Figure 1. Identification of Sec-containing MsrAs

(A) Multiple sequence alignment of MsrAs. Sec (U) residues are highlighted in red and the corresponding Cys residues are highlighted in blue. The conserved resolving Cys in C-terminal regions of Cys-containing MsrAs are shown in green. Cys55 and Cys95 of C. reinhardtii MsrA are highlighted in yellow. Accession numbers are indicated on the left. Only partial $A$. variegatum sequence is available. (B) Representative SECIS elements from $S$. fumaroxidans (bacterium, left), B. microplus (invertebrate animal, middle), and C. reinhardtii (green algae, right) selenoprotein MsrAs. Functionally important nucleotides in SECIS elements are highlighted in red. Sec codon (UGA) upstream of the bacterial SECIS element is highlighted in blue. Eukaryotic SECIS element structures were predicted with SECISearch (5) and the bacterial SECIS was predicted with RNAfold (20). (C) Phylogenetic analysis of Sec- (red) and Cys-containing (black) MsrAs. 


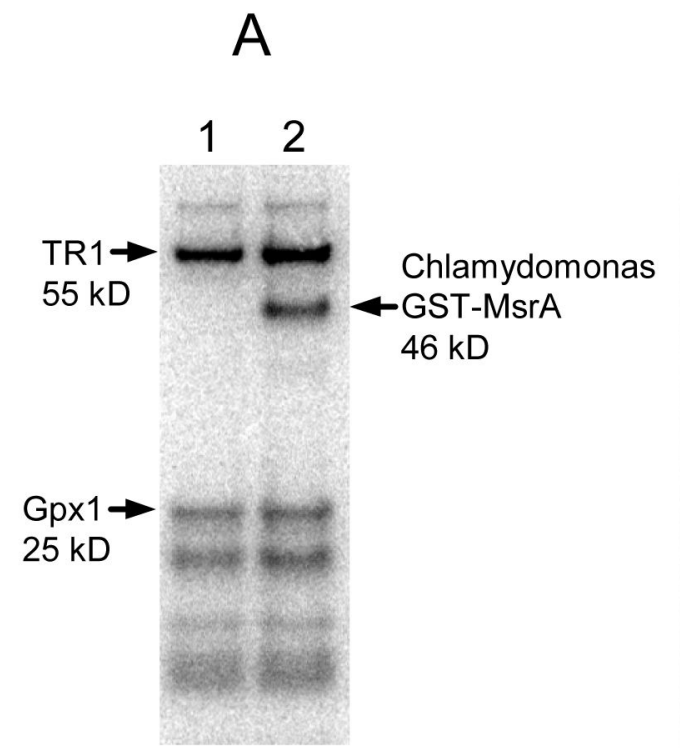

B

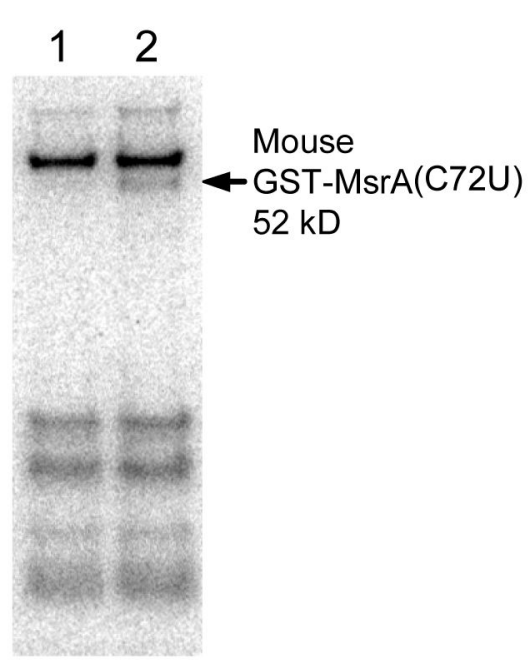

Figure $2 .{ }^{75}$ Se metabolic labeling of NIH 3 T3 cells expressing GST-fused Chlamydomonas MsrA (A) and GST-fusion selenoprotein form of mouse MsrA (B) The cells were transfected with an empty vector (lane 1) or GST-fusion constructs containing selenoprotein MsrAs (lane 2). The transfected cells were incubated for $24 \mathrm{~h}$ in the medium containing ${ }^{75} \mathrm{Se}$-selenite. The proteins were subjected to SDS-PAGE and the labeled proteins were visualized with a PhosphorImager. Endogenous selenoproteins thioredoxin reductase 1 $(\mathrm{TR} 1,55 \mathrm{kDa})$ and glutathione peroxidase $1(\mathrm{GPx} 1,25 \mathrm{kDa})$ are indicated on the left and the selenoproteins expressed from transfected constructs on the right. 


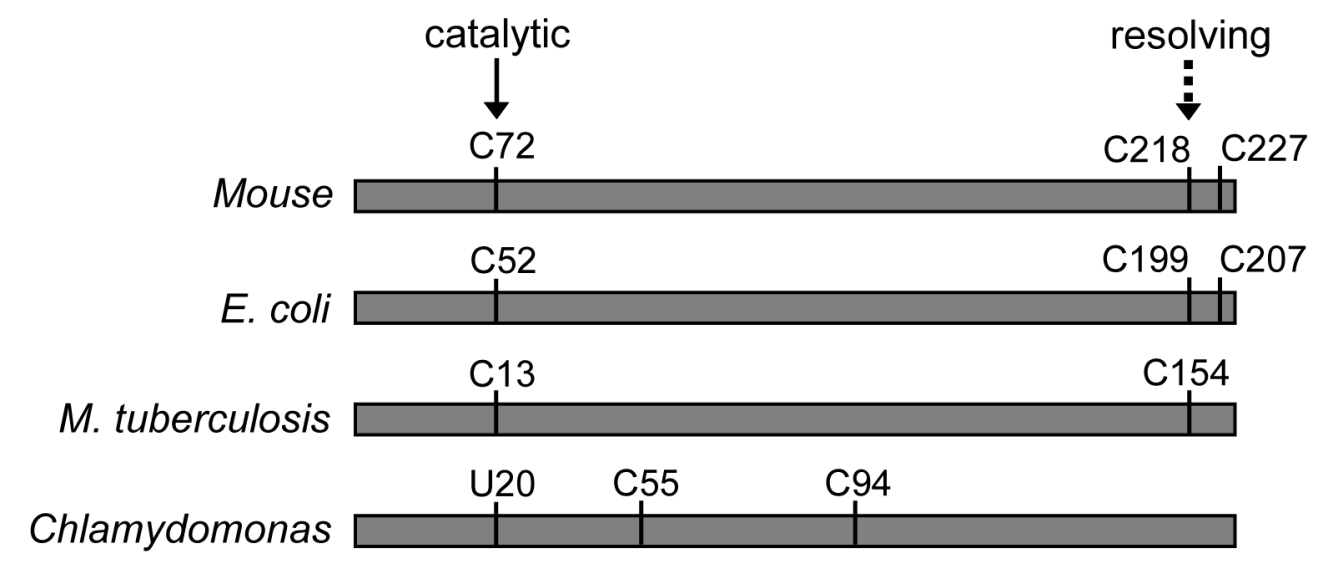

Figure 3. Schematic representation of MsrA sequences and location of resolving Cys Catalytic Cys or Sec (U) are indicated by an arrow. A resolving Cys that forms a disulfide bond with the catalytic Cys is indicated by a dashed arrow. Cys228 of mouse MsrA and Cys207 of $E$. coli MsrA are involved in the regeneration of the active enzymes by forming a disulfide with the resolving Cys. 


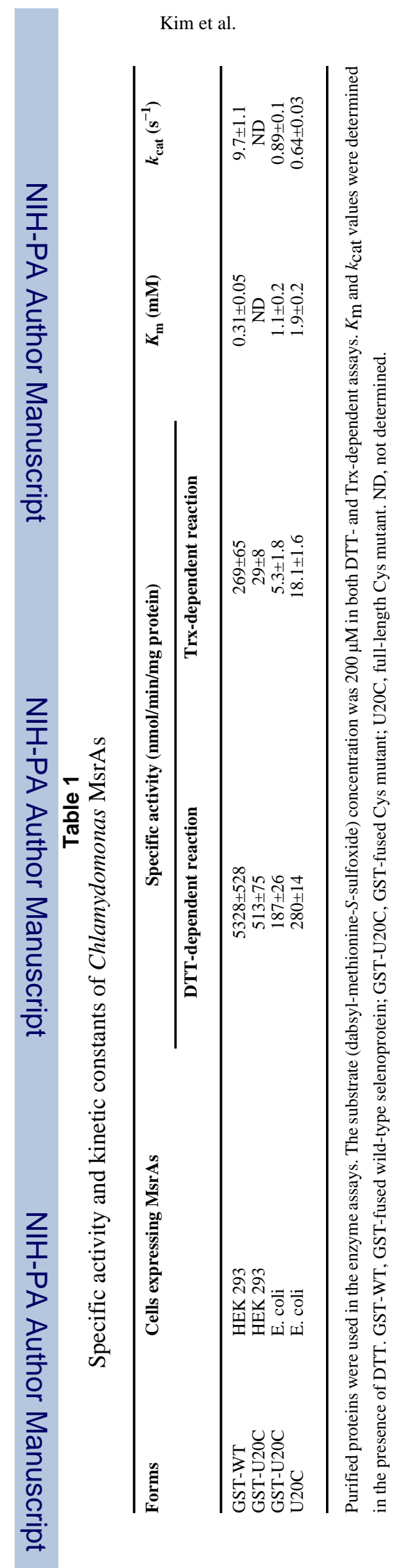

Page 15 


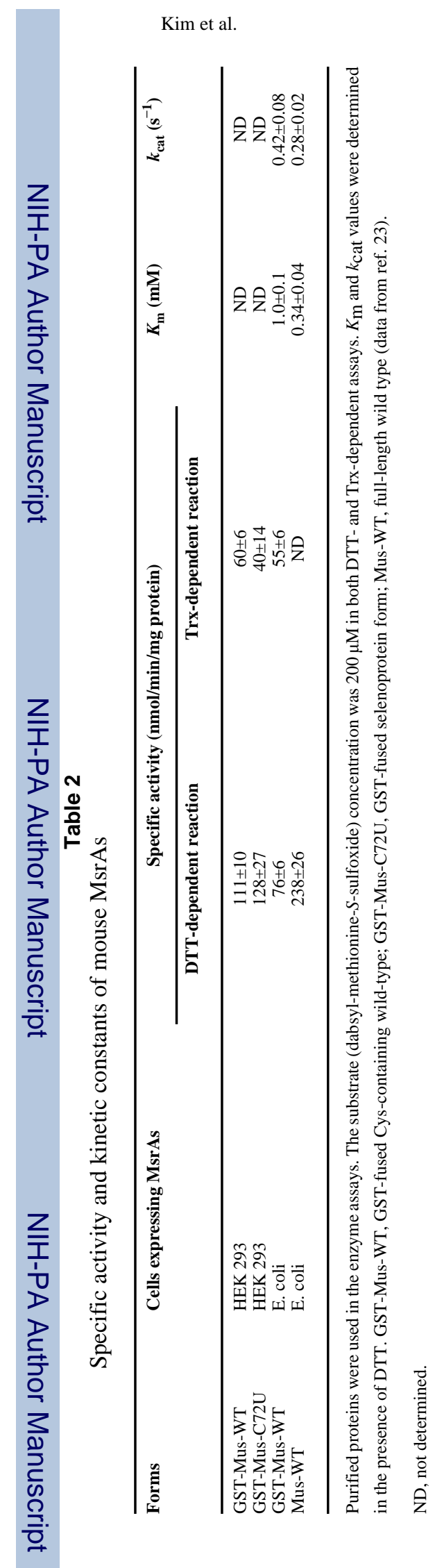

Page 16 
Table 3

Activity of Cys to Ser mutants of Chlamydomonas selenoprotein MsrA

\begin{tabular}{lcc}
\hline Forms & & Relative activity $(\%)$ \\
\cline { 2 - 3 } & DTT-dependent reaction & Trx-dependent reaction \\
\hline GST-WT & 100 & 100 \\
GST-C55S & 42 & 119 \\
GST-C94S & 105 & 114 \\
GST-C55S/C94S & 44 & 93 \\
\hline
\end{tabular}

Activities of the mutant proteins are shown relative to those of the wild-type form in both DTT- and Trx-dependent assays. The $100 \%$ activity corresponds to 3940 and $43 \mathrm{nmol} / \mathrm{min} / \mathrm{mg}$ protein in the DTT- and Trx-dependent assays, respectively. Crude extracts from transfected HEK 293 cells were used in the enzyme assays. Substrate (dabsyl-methionine-S-sulfoxide) concentration was $200 \mu \mathrm{M}$ in both the DTT-and Trx-dependent assays. All values are the average of two measurements and the difference between the measurements was less than $10 \%$. 
Table 4

Activity of Cys to Ser mutants of Cys- and Sec-containing forms of mouse MsrA

\begin{tabular}{llcc}
\hline Proteins & Forms & \multicolumn{2}{c}{ Relative activity (\%) } \\
\cline { 3 - 4 } & & DTT-dependent reaction & Trx-dependent reaction \\
\hline GST-fused Cys- & WT & 100 & 100 \\
containing & C218S & 6 & 7 \\
& C227S & 119 & 12 \\
GST-fused Sec- & C218S/C227S & 25 & 100 \\
containing & WT & 100 & 12 \\
& C218S & 170 & 72 \\
& C227S & 113 & 15 \\
\hline
\end{tabular}

Crude extracts of transfected HEK 293 cells were used in the enzyme assays. Substrate (dabsyl-methionine-S-sulfoxide) concentration was $200 \mu \mathrm{M}$ in both DTT- and Trx-dependent assays. The 100\% activities of Cys- and Sec-containing forms correspond to 63 and $86 \mathrm{nmol} / \mathrm{min} / \mathrm{mg}$ protein in the DTTdependent assay, respectively, and 30 and $13 \mathrm{nmol} / \mathrm{min} / \mathrm{mg}$ protein in the Trx-dependent assay, respectively. All values are the average of two measurements and the differences between the measurements were less than $15 \%$. 